\title{
CONDITIONS WHICH PRECLUDE THE EXISTENCE OF CRITICAL SOLUTIONS OF AN ORDINARY DIFFERENTIAL SYSTEM
}

\author{
L. P. BURTON
}

1. Introduction. Let $R$ be a region of $(n+1)$-dimensional space and let $R^{*}$ be a set such that $R \subset R^{*} \subset \bar{R}$ where $\bar{R}$ is the closure of $R$. Let the functions $f_{1}\left(x, y_{n}\right), \cdots, f_{k+1}\left(x, y_{k}\right), \cdots, f_{n}\left(x, y_{n-1}\right)$ be defined over $R^{*}$. All variables are assumed to be real. We shall consider the differential system,

$$
y_{i+1}^{\prime}=f_{i+1}\left(x, y_{i}\right) \quad(i=1, \cdots, n),
$$

where $y_{n+1}$ and $f_{n+1}\left(x, y_{n}\right)$ are by definition $y_{1}$ and $f_{1}\left(x, y_{n}\right)$ respectively. By a critical solution of (S) through a point $P:\left(x_{0}, y_{10}, \cdots, y_{n 0}\right)$ in $R^{*}$ is meant a solution $W(x): w_{1}(x), \cdots, w_{n}(x)$ which passes through $P$ and is such that for any other solution $Y(x): y_{1}(x), \cdots, y_{n}(x)$ which passes through $P$ and exists in $R^{*}$ with $W(x)$ over an interval $I:\left(x_{0}, x_{0}+a\right),\left(a^{2}>0\right)$, either $w_{k}(x) \leqq y_{k}(x)$ or $w_{k}(x) \geqq y_{k}(x)$ for each $k, 1 \leqq k \leqq n$, identically over $I$. The theory of critical solutions obviously has significance only in cases of nonunique solutions through $P$ and hereafter any mention of critical solutions will imply such nonuniqueness. W. Osgood [1] first proved the existence of critical solutions of $y^{\prime}=f(x, y)$. The theory of critical solutions was extended to the case $n>1$ by E. Kamke [2] and further extended jointly by W. M. Whyburn and the author [3]. The system (S) is a special case of more general systems considered by these writers and it will have critical solutions if it satisfies the conditions imposed by them. In the following it will be shown that under other conditions it is impossible for (S) to have critical solutions. Some related facts will also be established.

By the phrase "solution to the right of $P$ " shall be meant a solution existing over an interval $x_{0}<x<x_{0}+a, a>0$. Similarly, "solution to the left of $P$ " shall designate a solution existing over $x_{0}-a<x<x_{0}$, $a>0$. Throughout the ensuing discussion whenever the subscript $i$ appears it is to be understood that $i$ ranges over the set $(1, \cdots, n)$. The theory which shall be developed will be based on the prevalence

Presented to the Society, November 26, 1954 under the title Conditions which preclude the existence of maximum, minimum and minimax solutions of an ordinary differential system; received by the editors March 21, 1955 and, in revised form, July 18, 1955. 
of a certain set of conditions in $R^{*}$ for all $x>x_{0}$. There will, however, be a corresponding set of conditions whose existence in $R^{*}$ for all $x<x_{0}$ will lead to a dual theory. The situation for $x<x_{0}$ may be obtained from that for $x>x_{0}$ by means of the reflection $x=-t$. However, for the sake of clarity and completeness, the following device will be used: Each theorem will have a dual which will be designated by $*$. The statement and proof of the dual can be obtained from that of the theorem by reading "increasing" instead of "decreasing," "left" instead of "right," reversing the sense of all inequalities, except those restricting indices, and, in cases of reference to a theorem, replacing the designation of the theorem by that of its dual.

\section{Main theorems.}

Lemma. Let $\phi(x)$ and $\psi(x)$ be differentiable functions of $x$ over $I: x_{0}<x \leqq x_{0}+a, a>0$. Let $\psi(x)$ be such that $\lim _{x \rightarrow x_{0}} \psi(x)=y_{0}$ and let the set of values $[x, \phi(x)]$ have a limit point at $\left(x_{0}, y_{0}\right)$. If $\phi^{\prime}(x)<\psi^{\prime}(x)$ over I then $\phi(x)<\psi(x)$ over $I$.

Proof. Let $h(x)=\psi(x)-\phi(x)$. Then over $I, h^{\prime}(x)=\psi^{\prime}(x)-\phi^{\prime}(x)>0$. If $h(x)>0$ identically over $I$ there is nothing to prove. Otherwise, there exists a single value $c, x_{0}<c<x_{0}+a$ such that $h(c)=0$. For any value $d$ such that $x_{0}<d<c$ we have $h(d)<0$. By hypothesis there exists a sequence $\left\{x_{j}\right\}$ of values of $x$ which approaches $x_{0}$ as a limit and is such that $\lim _{x_{j} \rightarrow x_{0}} \phi\left(x_{j}\right)=y_{0}$. Then $\lim _{x_{j} \rightarrow x_{0}} h\left(x_{j}\right)=\lim _{x_{j} \rightarrow x_{0}} \psi\left(x_{j}\right)$ $-\lim _{x_{j} \rightarrow x_{0}} \phi\left(x_{j}\right)=0$. It follows that there exists a value $e, x_{0}<e<d$, for which $h^{\prime}(e)<0$. Thus $c$ cannot exist and the proof is complete.

THEOREMS 1 AND $1^{*}$. Let the functions $f_{i+1}\left(x, y_{i}\right)$ be defined over $R^{*}$ which contains $P:\left(x_{0}, y_{10}, \cdots, y_{n 0}\right)$. Let $y_{i}(x)$ and $z_{i}(x)$ be two distinct solutions of (S) existing in $R^{*}$ over $I: x_{0}<x<x_{0}+a, a>0$, and such that at least one approaches $P$ as a limit while the other has $P$ as a limit point. If over the subset of $R^{*}$ for which $x>x_{0}$ an odd number of the functions $f_{i+1}\left(x, y_{i}\right)$ are strictly decreasing in their $y$ variable and the remaining functions are strictly increasing in their $y$ variable, then it is impossible to have for any $k, 1 \leqq k \leqq n, y_{k}(x)-z_{k}(x)>0$ or $y_{k}(x)-z_{k}(x)<0$ identically over any subinterval $\bar{I}: x_{0}<x<x_{0}+b \leqq x_{0}+a$ of $I$.

Proof. Assume the conclusion is not true and with no loss of generality assume both that $k=1$ and $y_{1}(x)>z_{1}(x)$ over $\bar{I}$. It follows that, over $\bar{I}, y_{2}^{\prime}(x)>z_{2}^{\prime}(x)$ in case $f_{2}\left(x, y_{1}\right)$ is strictly increasing in $y_{1}$ or $y_{2}^{\prime}(x)<z_{2}^{\prime}(x)$ in the opposite case. Correspondingly, by the lemma, $y_{2}(x)>z_{2}(x)$ or $y_{2}(x)<z_{2}(x)$ uniformly over $\bar{I}$. One now sees by induction that either $y_{i}(x)>z_{i}(x)$ or $y_{i}(x)<z_{i}(x)$ uniformly over $\bar{I}$. If $f_{j+1}\left(x, y_{j}\right)$ is increasing in $y_{j}$ we have 


$$
\left(y_{j+1}-z_{j+1}\right)\left(y_{j}-z_{j}\right)>0 \text { over } \bar{I}
$$

and the sense of the inequality is reversed if $f_{j+1}\left(x, y_{j}\right)$ is decreasing in $y_{j}$. Since an odd number of the $f_{i+1}\left(x, y_{i}\right)$ are strictly decreasing in their $y$ variable the above inequality changes sign an odd number of times as $j$ ranges from 1 to $n$. Hence, forming the product of the expressions (1),

$$
\begin{aligned}
\left(y_{n+1}-z_{n+1}\right)\left(y_{n}-z_{n}\right)^{2} \cdots \cdot\left(y_{2}-z_{2}\right)^{2}\left(y_{1}-z_{1}\right) & \\
& =\left(y_{n}-z_{n}\right)^{2} \cdots \cdot\left(y_{1}-z_{1}\right)^{2}<0
\end{aligned}
$$

and this contradiction completes the proof.

Corollary 1. Let $\bar{x}_{0} \geqq x_{0}$ be the smallest value for which $Q:\left(\bar{x}_{0}\right.$, $\left.y_{10}, \cdots, y_{n 0}\right)$ is a bifurcation point of the solutions $y_{i}(x)$ and $z_{i}(x)$. Then for any $k, 1 \leqq k \leqq n, y_{k}(x)-z_{k}(x)$ has an infinite number of zeros in any interval $\bar{x}_{0}<x<\bar{x}_{0}+a, a>0$.

REMARK. If $y_{i 0}=0$ and if $y_{i}(x)$ is the identically vanishing solution, then this corollary implies that $z_{i}(x)$ oscillates infinitely many times in any interval $\bar{x}_{0}<x<\bar{x}_{0}+\epsilon, \epsilon>0$. If, further, the solution $z_{i}(x)$ has $Q:\left(\bar{x}_{0}, 0, \cdots, 0\right)$ as a limit then $z_{i}(x)$ is the type of solution which is said by many writers to be "self-oscillatory." Such solutions are, of course, very important in dynamical considerations.

CoROllary 2. Let (S) and $R$ be such that for $x>x_{0}$ and for some $k$, $1 \leqq k \leqq n, f_{k}\left(x, y_{k-1}\right)$ is uniformly greater or uniformly less in $R$ than in $R^{*}-R$. If $(\mathrm{S})$ has one solution $y_{i}(x)$ in $R^{*}-R$ which passes through $P$ then every solution in $R^{*}$ which has a limit point at $P$ is in $R^{*}-R$.

Proof. Let $z_{i}(x)$ be a solution in $R$ having $P$ as a limit point. Then in $R^{*}$ for $x>x_{0}$ either $z_{k}^{\prime}(x)>y_{k}^{\prime}(x)$ or $z_{k}^{\prime}(x)<y_{k}^{\prime}(x)$. By the lemma, therefore, either $z_{k}(x)>y_{k}(x)$ or $z_{k}(x)<y_{k}(x)$ in $R^{*}$ over $I$. This contradicts the theorem and proves that $z_{i}(x)$ cannot exist.

COROLlaRy 3. If $y_{i}(x)$ and $z_{i}(x)$ are such that, over $I, y_{k}(x) \equiv z_{k}(x)$ for some $k, 1 \leqq k \leqq n$, then $y_{i}(x) \equiv z_{i}(x)$ over $I$.

Proof. From $y_{k+1}^{\prime}(x)=z_{k+1}^{\prime}(x)$ one obtains $y_{k+1}(x) \equiv z_{k+1}(x)$ over $I$, since the constant of integration is zero. By induction, $y_{i}(x) \equiv z_{i}(x)$ over $I$.

Theorems 2 AND 2*. The system (S), under the hypotheses of Theorem 1, can have no critical solution existing in $R$ through and to the right of $P$.

Proof. Suppose there is such a critical solution, $w_{i}(x)$, and let $y_{i}(x)$ be any other solution existing in common with $w_{i}(x)$ in $R$ through and to the right of $P$ over $x_{0}<x<x_{0}+d, d>0$. Then for 
some $k, 1 \leqq k \leqq n, y_{k}(x) \not w_{k}(x)$ over $\left(x_{0}, x_{0}+d\right)$. Let $c, x_{0}<c<x_{0}+d$, be such that $y_{k}(c) \neq w_{k}(c)$. There exists a largest value $b, x_{0}<b<c$, for which $w_{k}(b)=y_{k}(b)$. Notice that if for any $j, 1 \leqq j \leqq n$, the conditions $y_{j}(b)=w_{j}(b), y_{j}^{\prime}(b) \neq w_{j}^{\prime}(b)$ hold, then the assumption that $w_{i}(x)$ is a critical solution is contradicted. Therefore, $y_{k}^{\prime}(b)=w_{k}^{\prime}(b)$ and furthermore, since $f_{k}\left(x, y_{k-1}\right)$ is strictly increasing or decreasing in $y_{k-1}$, one sees that $y_{k-1}(b)=w_{k-1}(b)$. By a straightforward inductive proof, it follows that $y_{i}(b)=w_{i}(b)$. This implies that there are two distinct solutions of $(\mathrm{S})$ through the point $\left[b, w_{1}(b), \cdots, w_{n}(b)\right]$ such that, over the interval $b<x<c, w_{k}(x)-y_{k}(x)$ does not vanish. This contradicts Theorem 1. Therefore, there can be no critical solution over $x_{0}<x<x_{0}+d$.

\section{Applications to the case $n=1$.}

Theorems 3 And $3 *$. Let $P$ be a point in $R:(x, y)$, let $f(x, y)$ be continuous in $R$ and strictly decreasing in $y$ for each fixed $x>x_{0}$. Then $y^{\prime}=f(x, y)$ has a unique solution existing in $R$ to the right of $x_{0}$.

Proof. The hypotheses are sufficient to insure the existence of at least one solution through and to the right of $P$. If this solution were not unique, there would exist critical solutions through and to the right of $P[1]$. This contradicts Theorem 2 .

Theorems 4 ANd $4^{*}$. Let $y^{\prime}=f(x, y)$ satisfy the hypotheses of Theorem 1 . Let $y(x)$ be a solution which exists in $R$ to the right of $x_{0}$ and which approaches $P:\left(x_{0}, y_{0}\right)$ as a limit. Then there can exist no other solution $u(x)$ in $R^{*}$ to the right of $P$ which has $P$ as a limit point.

Proof. By Theorem 1, $y(x)-u(x)=0$ for some value in any interval $x_{0}<x<x_{0}+a, a>0$. Let $\theta$ be such a value. Then, $[\theta, y(\theta)]$ lies in the interior of $R$. By Theorem 3 , it follows that $y(x) \equiv u(x)$ over $\theta \leqq x<\theta+a$. Since $\theta$ may be chosen arbitrarily close to $x$, we have $y(x) \equiv u(x)$ over $x_{0}<x<x_{0}+a$.

REMARK. This simple uniqueness theorem does not seem to appear in the literature. A similar theorem, but one with more restrictive hypotheses, has been presented by E. J. McShane [4].

4. Examples. (a) $y^{\prime}=-(y / x)^{1 / 3}$. It is immediately seen that $-(y / x)^{1 / 3}$ is decreasing in $y$ for each fixed $x>0$. By Theorem 3 there exists a unique solution through and to the right of $(a, 0),(a>0)$. This solution is obviously $y \equiv 0$. By Theorem $3^{*}$ there may exist more than one solution to the left of $(a, 0) \cdot y^{2}=\left(a^{2 / 3}-x^{2 / 3}\right)^{3}$ defines a pair of such solutions. On the other hand, if $a<0$, the solution is unique to the left of $(a, 0)$ and nonunique to the right. By Theorems 
4 and $4^{*}, y \equiv 0$ is the only solution through $(0,0)$. Similar arguments can be made for the inverse equation $x^{\prime}=-(x / y)^{1 / 3}$. Thus the behavior of the family $x^{2 / 3}+y^{2 / 8}=a^{2 / 3}$ of four-cusped hypocycloids is virtually described by the equation $y^{\prime}=-(y / x)^{1 / 3}$ in the light of Theorems 3 and $3 *, 4$ and $4^{*}$.

(b) $y^{\prime}=-\sin x y^{1 / 3}$. Let $R$ be the square $-1<x<1,-1<y<1$. By Theorems 3 and $3^{*}$ this equation can have only the identically vanishing solutions through $(0,0)$ in $R$. The equation $y^{\prime}=-\sin x y^{2 / 3}$, on the other hand, cannot have a solution in the open upper-half plane, but may have nonunique solutions through $(0,0)$ and extending into the lower-half plane.

(c) $y_{1}^{\prime}=y_{2}, y_{2}^{\prime}=-y_{1}^{1 / 2}$. Let $R$ be defined by $-\infty<x<\infty, 0<y_{1}<\infty$, $-\infty<y_{2}<\infty$. Since $y_{1} \equiv y_{2} \equiv 0$ is a solution in $\bar{R}-R$ it follows by Corollary 2 of Theorem 1 that every solution having a limit point at $(0,0,0)$ is in the plane $y_{1}=0$. By Corollary 3 one concludes that $y_{1} \equiv y_{2} \equiv 0$ is the unique solution having a limit point at $(0,0,0)$.

(d) $y_{1}^{\prime}=\left(-1 / x^{2}\right) y_{2}, y_{2}^{\prime}=\left(1 / x^{2}\right) y_{3}, y_{3}^{\prime}=\left(1 / x^{2}\right) y_{1}$. Let $R$ be the set of all points $\left(x, y_{1}, y_{2}, y_{3}\right)$ having $x>0$. The solution $y_{1} \equiv y_{2} \equiv y_{3} \equiv 0$ lies in $R$ and has the point $P:(0,0,0,0)$ in $\bar{R}$ as a limit. According to the remark following Corollary 1 to Theorem 1 , any other solution having $P$ as a limit point must oscillate infinitely many times in any arbitrarily small interval to the right of the point of bifurcation. Such a solution (with $P$ as the bifurcation point) is exhibited by

$$
\begin{aligned}
& y_{1}=e^{(-1 / 2 x)} \cos \left[3^{1 / 2} /(2 x)\right], \\
& y_{2}=(-1 / 2) e^{(-1 / 2 x)}\left\{3^{1 / 2} \sin \left[3^{1 / 2} /(2 x)\right]+\cos \left[3^{1 / 2} /(2 x)\right]\right\}, \\
& y_{3}=(1 / 2) e^{(-1 / 2 x)}\left\{-3^{1 / 2} \sin \left[3^{1 / 2} /(2 x)\right]+\cos \left[3^{1 / 2} /(2 x)\right]\right\} .
\end{aligned}
$$

\section{REFERENCES}

1. W. Osgood, Beweis der Existenz einer Losung der Differentialgleichung $d y / d x$ $=f(x, y)$ ohne Hinzunahme der Cauchy-Lipschitzschen Bedingung, Monatshefte für Mathematik und Physik vol. 9 (1898) pp. 341-345.

2. E. Kamke, Zur Theorie der Systems gewohnlicher Differentialgleichungen, Acta Math. vol. 58 (1932) pp. 57-85.

3. L. P. Burton and W. M. Whyburn, Minimax solutions of ordinary differential systems, Proc. Amer. Math. Soc. vol. 5 (1952) pp. 794-803.

4. E. J. McShane, Integration, Princeton University Press, 1944, p. 348.

Alabama Polytechnic Institute 